\title{
Flight Loads Analysis of a Maneuvering Transport Aircraft
}

\author{
Hui Zhang ${ }^{1, a}$, Jie $\mathrm{Li}^{1, \mathrm{~b}}$, Qiong Liu ${ }^{2, \mathrm{c}}$ \\ ${ }^{1}$ School of Aeronautics, Northwestern Polytechnical University, China \\ ${ }^{2}$ Universidad Politécnica de Madrid, Spain \\ azhanghui_0104@126.com, 'blijieruihao@163.com, 'cqiong@torroja.dmt.upm.es
}

Keywords: flight load; dynamic derivative; dynamic maneuvers; doublet lattice method; control surface

\begin{abstract}
The paper provides a method applicable for the determination of flight loads for maneuvering aircraft, in which aerodynamic loads are calculated based on doublet lattice method, which contains three primary steps. Firstly, non-dimensional stability and control derivative coefficients are obtained through solving unsteady aerodynamics in subsonic flow based on a doublet lattice technical. These stability and control derivative coefficients are used in second step. Secondly, the simulation of aircraft dynamic maneuvers is completed utilizing fourth order Runge-Kutta method to solve motion equations in different maneuvers to gain response parameters of aircraft due to the motion of control surfaces. Finally, the response results calculated in the second step are introduced to the calculation of aerodynamic loads. Thus, total loads and loads distribution on different components of aircraft are obtained. According to the above method, abrupt pitching maneuvers, rolling maneuvers and yawing maneuvers are investigated respectively.
\end{abstract}

\section{Introduction}

Flight loads including aerodynamic loads and inertia loads are the original data of aircraft structure design. Only under the rather accurate structure loads the designed aircraft can satisfy the given aircraft criteria such as flight quality criteria, strength criteria. Therefore the accurate prediction of structure loads is a key factor for aircraft structure design which impacts directly on the weight of a aircraft, the capacity of maneuverability and the safety of flight.

When a pilot manipulates the control surface to make it rotate according to an expecting rule, the response of aircraft such as pitch, roll or yaw will start. To determine loads during maneuvers, the flight mechanics equations of motion need to be solved to obtain the response characteristics of the aircraft. On the other hand, it is necessary to consider the flexible effects on maneuver loads calculation, especially for more highly flexible aircraft ${ }^{[1,2,3]}$. Thus structural mechanics equations have to be taken into account simultaneously. Therefore the accurate prediction of maneuver flight loads need to simulate maneuvering aircraft by aerodynamic, flight mechanics, and structural mechanics coupling ${ }^{[4,5]}$. However these advanced simulation method may be complex and involve the use of large digital computers, and a mass of calculation time has to be cost for a maneuvering flight simulation on the current computer condition. Moreover, the magnitude of the number of load points and conditions may be investigated for the aircraft structure strength design, leading to the tremendous difficulty in determining the loads for maneuvering aircraft by completely using the coupled method. In fact, it is the most cost time to solve Euler/Navier-Stokes to obtain the unsteady aerodynamic loads in the coupled method. If this portion of calculation time can be reduced, the above difficulty will be overcome. The calculation time of solving unsteady aerodynamic loads can be greatly reduced based on doublet lattice method ${ }^{[6,7]}$. Of course, the accuracy of solution based on doublet lattice method will drop compare with Euler/ Navier-Stokes. Whereas the accuracy of 
solution can satisfy the engineering requirement in initial aircraft design stages. In addition, even for the detailed design stage the maneuver loads can be determined through doublet lattice method firstly then validating the results from Euler/ Navier-Stokes equations.

The method discussed in the paper is applicable for the determination of flight loads for maneuvering aircraft, in which aerodynamic loads are calculated based on doublet lattice method.

\section{Dynamic Derivative Computations}

The definitions of longitudinal stability derivatives are given in the equations for the lift coefficient

$$
C_{z}=C_{z_{0}}+C_{z_{\alpha}} \alpha+C_{z_{\dot{\alpha}}} \frac{\dot{\alpha} c}{2 V}+C_{z_{q}} \frac{q c}{2 V}
$$

and moment coefficient

$$
C_{m}=C_{m_{0}}+C_{m_{\alpha}} \alpha+C_{m_{\alpha}} \frac{\dot{\alpha} c}{2 V}+C_{m_{q}} \frac{q c}{2 V}
$$

Harmonic plunging with amplitude $h_{0}$ is considered, then $\alpha=\frac{h_{0}}{V} i \omega e^{i \omega t}$, and Eq.1 and Eq.2 become

$$
\begin{aligned}
& C_{z}=\frac{2 h_{0}}{c}\left(i k C_{z_{\alpha}}-k^{2} C_{z_{\dot{\alpha}}}\right) e^{i \omega t} \\
& C_{m}=\frac{2 h_{0}}{c}\left(i k C_{m_{\alpha}}-k^{2} C_{m_{\dot{\alpha}}}\right) e^{i \omega t}
\end{aligned}
$$

In addition, based on a doublet lattice technical one can obtain $C_{z}=\bar{C}_{z} e^{i \omega t}$ and $C_{m}=\bar{C}_{m} e^{i \omega t}$, Thus

$$
\begin{aligned}
& \bar{C}_{z}=\frac{2 h_{0}}{c}\left(i k C_{z_{\alpha}}-k^{2} C_{z_{\dot{\alpha}}}\right) \\
& \bar{C}_{m}=\frac{2 h_{0}}{c}\left(i k C_{m_{\alpha}}-k^{2} C_{m_{\dot{\alpha}}}\right)
\end{aligned}
$$

Similarly, if harmonic pitching with amplitude $\alpha_{0}$ is next considered, one can obtain

$$
\begin{aligned}
& \bar{C}_{z}=\alpha_{0}\left[C_{Z_{\alpha}}+i k\left(C_{z_{\alpha}}+C_{Z_{q}}\right)\right] \\
& \bar{C}_{m}=\alpha_{0}\left[C_{m_{\alpha}}+i k\left(C_{m_{\dot{\alpha}}}+C_{m_{q}}\right)\right]
\end{aligned}
$$

Obviously, combining Eq.5 through Eq.8 and the results from a doublet lattice technical, longitudinal stability derivatives will be obtained.

The calculation of lateral-directional derivatives is similar to that for the longitudinal derivatives illustrated above, in which harmonic sideslip roll and yaw motions are respectively considered. In the same way, control surfaces stability derivatives with their angle velocity will be determined through harmonic motions of control surfaces.

\section{Dynamic Maneuvers}

The equations of motion for both pitching maneuvers and rolling maneuvers are derived from the work of Jan R. Wright and Jonathan E. Cooper in Ref.1, which are repeated respectively as follow.

$$
\left[\begin{array}{cc}
m & 0 \\
0 & I_{y}
\end{array}\right]\left\{\begin{array}{c}
\dot{w} \\
\dot{q}
\end{array}\right\}+\left[\begin{array}{cc}
0 & -m U_{e} \\
0 & 0
\end{array}\right]\left\{\begin{array}{l}
w \\
q
\end{array}\right\}-\left[\begin{array}{cc}
Z_{w} & Z_{q} \\
M_{w} & M_{q}
\end{array}\right]\left\{\begin{array}{l}
w \\
q
\end{array}\right\}=\left\{\begin{array}{l}
Z_{\delta_{z}} \\
M_{\delta_{z}}
\end{array}\right\} \delta_{z}
$$


Where, $\quad Z_{w}=\frac{1}{2} \rho V S C_{z \alpha}, \quad Z_{q}=\frac{1}{4} \rho V S c C_{Z q}, \quad Z_{\delta_{z}}=\frac{1}{2} \rho V^{2} S C_{z \delta_{z}}, \quad M_{w}=\frac{1}{2} \rho V S c C_{M \alpha}, \quad M_{q}=\frac{1}{4} \rho V S c^{2} C_{M q}$, $M_{\delta_{z}}=\frac{1}{2} \rho V^{2} S c C_{M \delta_{z}}$.

$$
I_{\chi} \dot{p}-L_{p} p=L_{\delta_{a}} \delta_{a}
$$

Where, $L_{p}=\frac{1}{4} \rho V S b^{2} C_{L p}, \quad L_{\delta_{a}}=\frac{1}{2} \rho V^{2} S b C_{L \delta_{a}}$.

Eq.9 may be solved to determine the response corresponding to a particular elevator input, and Eq.10 may be used to determine the response to any aileron input.

The equations of motion for yawing maneuvers are derived from Ref.2.

$$
\left\{\begin{array}{l}
\frac{d \beta}{d t} \\
\frac{d r}{d t}
\end{array}\right\}=\left[\begin{array}{ll}
Y_{\beta} & Y_{r} \\
N_{\beta} & N_{r}
\end{array}\right]\left\{\begin{array}{l}
\beta \\
r
\end{array}\right\}+\left[\begin{array}{c}
Y_{\delta_{r}} \\
N_{\delta_{r}}
\end{array}\right] \delta_{r}
$$

Where, $\quad Y_{\beta}=\frac{g q S_{W}}{W V_{\infty}} C_{Y}^{\beta}, \quad Y_{r}=\frac{g q S_{W}\left[L_{v}(\beta)+\Delta x_{C G}\right]}{W V_{\infty}^{2}} C_{Y}^{\beta}+1, \quad N_{r}=\frac{q S_{W} b^{2}}{2 I_{z} V_{\infty}} C_{n}^{r}+q S_{W} \frac{\left(L_{v}(\beta)+\Delta x_{C G}\right) c_{A W}}{V_{\infty} I_{z}} C_{Y, v}^{\beta}$, $Y_{\delta_{r}}=\frac{g q S_{W}}{W V_{\infty}} C_{Y}^{\delta_{r}}, N_{\delta_{r}}=\frac{q S_{W} b}{I_{z}} C_{n}^{\delta_{r}}+\frac{q S_{W}\left(L_{v}\left(\delta_{r}\right)+\Delta x_{C G}\right)}{I_{z}} C_{Y}^{\delta_{r}}$.

Eq.11 may be used to determine the response to any rudder input.

Eq.9 to Eq.11 are solved by using fourth order Runge-Kutta method to determine the time history of aircraft load factor and related parameters such as pitch velocity, roll velocity, yaw velocity, angle of attack, roll angle and side angle.

\section{The Determination of Flight Loads for Maneuvering Aircraft}

According to the method discussed in the paper, the calculation of flight loads for a maneuvering aircraft is demonstrated as follows.

Description of the Calculation Model. The aerodynamic model is given in Fig.1, which includes the reference chord of $2.9 \mathrm{~m}$, span of $24.5 \mathrm{~m}$, Wing, horizontal tail and vertical tail idealized as lifting surfaces, while fuselage idealized as slender body and interference body. Non-dimensional stability and control derivative coefficients are calculated at Mach number of 0.30 , the dynamic pressure of $6372 \mathrm{~Pa}$, and listed in Table 1 . The total weight of the aircraft is $22000 \mathrm{~kg}$, the centre of gravity is $1.2 \mathrm{~m}$ forward of the intersection of the fuselage and wing elastic axis, and the centroidal moment of inertia in pitch, roll and yaw are $294111 \mathrm{~kg} \cdot \mathrm{m}^{2}, 251036 \mathrm{~kg} \cdot \mathrm{m}^{2}$ and $534423 \mathrm{~kg} \cdot \mathrm{m}^{2}$ respectively.

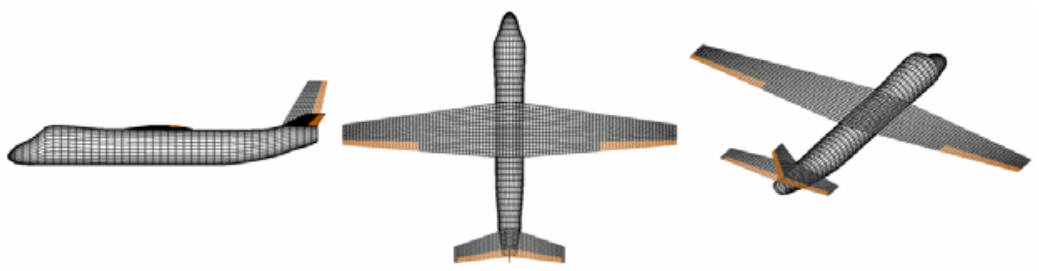

Fig.1 Aerodynamic model

Abrupt Pitch Maneuver. For abrupt pitch maneuver, the initial condition is Steady level flight, in which load factor is 1 . After airplane is trimmed, elevator is deflected with the maximum available rate, and the deflect angle is determinate when load factor becomes the design value in the whole maneuver. The input of elevator angle depicted in Fig.2 (a) is applied to the airplane, and the 
response results of pitch rate, angle of attack, and load factor are also described in Fig.2 (a). The corresponding aerodynamic loads on different components of aircraft variation with time are given in Fig.3 (a). The severe load states of components of airplane on the above condition can be obtained. Because abrupt pitch maneuver is often the critical load condition of horizontal tail, elevator and aft fuselage, their maximum load values have to be considered here. Horizontal tail load gets a negative maximum value, $-60470 \mathrm{~N}$, at 0.44 second, and a plus maximum value, $75654 \mathrm{~N}$, at 0.89 second. The results indicate that horizontal tail load change extensively in abrupt pitch maneuver, even with contrary directions. In addition, elevator and fuselage load reach the maximum value, $-52483 \mathrm{~N}$ and $59244 \mathrm{~N}$, at 0.44 and 0.87 second respectively.

Table 1 Non-dimensional stability and control derivative coefficients

\begin{tabular}{|c|c|c|c|c|c|}
\hline$C_{Z \alpha}$ & -4.8947 & $C_{L \beta}$ & -0.06693 & $C_{N r}$ & -0.1204 \\
\hline$C_{M \alpha}$ & -2.3830 & $C_{N \beta}$ & 0.1469 & $C_{Y \delta_{r}}$ & -0.2756 \\
\hline$C_{Z q}$ & -12.6625 & $C_{Y p}$ & -0.06595 & $C_{L \delta_{r}}$ & -0.03421 \\
\hline$C_{M q}$ & -20.2577 & $C_{L p}$ & -0.4369 & $C_{N \delta_{r}}$ & 0.1363 \\
\hline$C_{z \delta_{z}}$ & -0.5748 & $C_{N p}$ & -0.00783 & $C_{Y \delta_{a}}$ & -0.03938 \\
\hline$C_{M \delta_{z}}$ & -1.6286 & $C_{Y r}$ & 0.4512 & $C_{L \delta_{a}}$ & -0.2842 \\
\hline$C_{Y \beta}$ & -0.4346 & $C_{L r}$ & 0.0227 & $C_{N \delta_{a}}$ & -0.002429 \\
\hline
\end{tabular}

Roll Maneuver. For roll maneuver, the ailerons are deflected with the maximum available rate in roll maneuver. When the prescribed angle of roll is reached, the ailerons return to stop the maneuver. The input of aileron angle depicted in Fig.2 (b) is applied to the airplane, the response results of roll rate, angle of roll are described in Fig.2 (b). The corresponding aerodynamic loads on wing and aileron of aircraft variation with time are given in Fig.3 (b). Time history of aerodynamic loads on wing and aileron show that right wing load become minimum when left wing load reach the maximum value, and that aileron behave the same process as wing. Right wing load is 69799N when left wing load reach $154040 \mathrm{~N}$, and right wing load is $159670 \mathrm{~N}$ when left wing become 64172 N. Maximum left aileron load is $22424 \mathrm{~N}$ when right one is $-15415 \mathrm{~N}$.

Yaw Maneuver. For yaw maneuver the rudder is deflected with the maximum available rate in yaw maneuver. When the steady angle of side is reached, the rudder returns to stop the maneuver. The input of rudder angle depicted in Fig.2 (c) is applied to the airplane, the response results of angle of side and lateral acceleration are described in Fig.2 (c). The corresponding aerodynamic loads on vertical tail and rudder of aircraft variation with time are given in Fig.3 (c). The maximum loads of vertical tail and rudder are respectively $19514 \mathrm{~N}$ and $10700 \mathrm{~N}$.

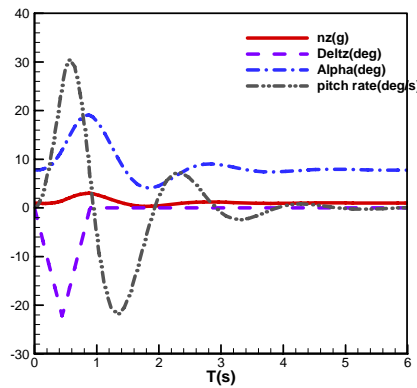

(a) Abrupt pitch maneuver

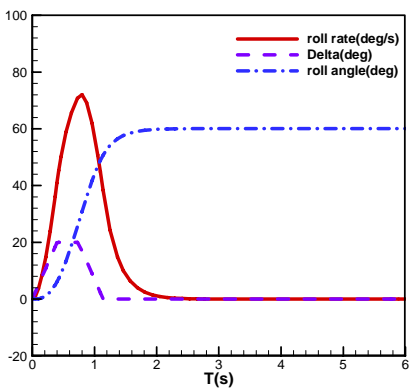

(b) Roll maneuver

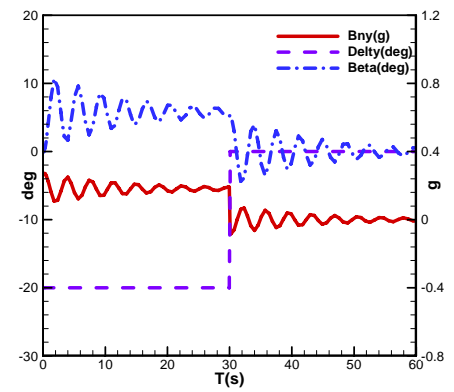

(c) Yaw maneuver

Fig.2 Response parameters variation with time 


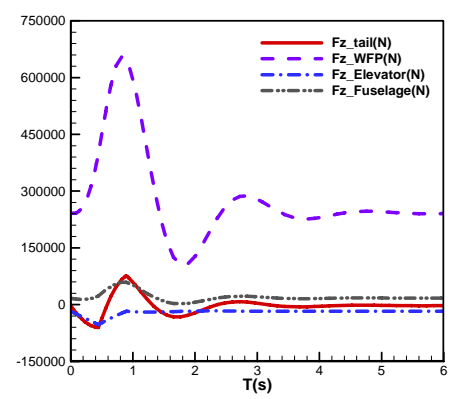

(a) Abrupt pitch maneuver

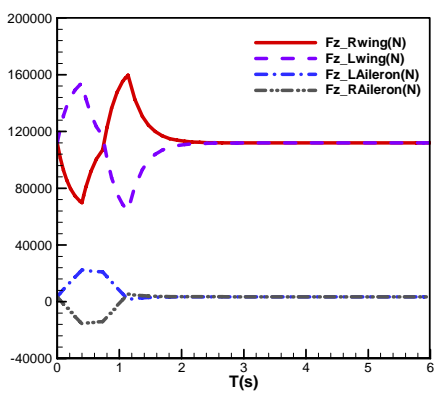

(b) Roll maneuver

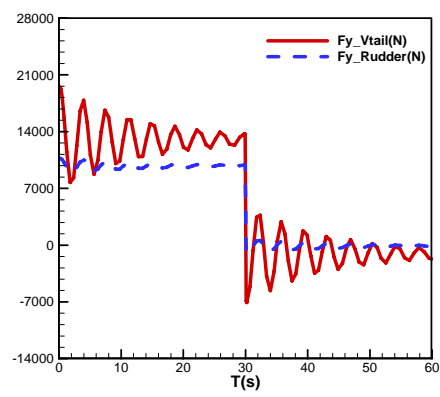

(c) Yaw maneuver

Fig.3 Aerodynamic loads on different components of aircraft variation with time

\section{Summary}

Flight loads on different components of airplane in different maneuver can be obtained quickly and effectively using the method introduced in the paper. Subsequently the severe load states of components of airplane are found, which should be considered in structure design. And the distribution of flight loads on components of airplane acquired can be directly applied to the finite model in intensity check of aircraft.

In addition, the current researches only concern the calculation of flight loads for rigid model. Actually, aircraft elasticity has an important impact on analysis results and the distribution of flight loads. Accordingly, the calculation of flight loads for flexible model will be carried out subsequently.

\section{Acknowledgments}

This work was supported by the National Science Foundation of China (Grant No. 90816027, 10772148, 11172240,). The authors would like to thank Professor LI Fengwei for many insightful technical discussions.

\section{References}

[1] Jan R. Wright, and Jonathan E. Cooper, "Introduction to Aircraft Aeroelasticity and Loads”, John Wiley \& Sons, Ltd, 2007, 266-269, 279-280.

[2] Aircraft Design Manual, the 9th Volume: Loads Strength and Stiffness, Bei Jing: Aviation Industry Publishing House, 2001, 28-30.

[3] Lomax, T.L. "Structure Loads Analysis for Commercial Transport Aircraft: Theory and practice”, AIAA Education Series, 1996.

[4] Andreas Schutte, Gunnar Einarsson. "Numerical Simulation of Maneuvering Aircraft by Aerodynamic, Flight Mechanics, and Structure Mechanics Coupling”, Journal of Aircraft, Vol.46, No. 1, 2009.

[5] Li Xile, Yang Yong, "Numerical Simulation of the Free Rolling Motion of a Delta Wing Configuration with Aileron Deflection”, Acta Aeronautica et Astronautica Sinica, Vol.33, No.3, 2012.

[6] Gray, W.L, and Schenk, K.M., “A method for Calculating the Subsonic Steady-State Loading on an Airplane Wing of Arbitrary Plan Form and Stiffness,” NACA TN 3030,Dec. 1953.

[7] Albino, E. and Rodden, W.P. "A doublet-lattice Method for Calculating lift distribution on oscillating surfaces in subsonic flows. AIAA Journal, 7(2). 